\title{
Mens: \\ दोis \\ FrAD \\ Effet à long terme de la monoculture sur le rendement en grain du maïs (Zea mays $L$ ) en conditions non irriguées
}

Agronomie

\author{
E Lubet, D Plénet ${ }^{\star}$, C Juste \\ INRA, station d'Agronomie, centre de recherches de Bordeaux, BP 81, F33883 Villenave-d'Ornon cedex, France
}

(Reçu le 15 octobre 1992; accepté le 6 juillet 1993)

\begin{abstract}
Résumé - Dans 2 dispositifs expérimentaux situés sur sol limono-sableux des Landes de Gascogne, on a mesuré, durant 21 et 24 années respectivement, les rendements d'une monoculture non irriguée de maïs grain comportant les modalités suivantes : monoculture avec (TR) ou sans (TE) restitution des résidus de récolte. Introduction d'une interculture d'engrais vert à base de ray-grass d'Italie, semé en août et enfoui au printemps en même temps que les résidus de récolte $(R G)$. Interruption tous les 4 ans de la monoculture par une prairie temporaire de 3 ans (PT). Malgré de grandes variations interannuelles dues aux fluctuations du déficit hydrique estival, on montre que la culture continue du maïs ne provoque pas de baisse du rendement en grain de la culture. On enregistre, au contraire, une tendance à leur accroissement au cours du temps (0,76 et 1,19 q/ha/an dans chacun des 2 dispositifs), en raison vraisemblablement de l'amélioration génétique du matériel végétal. L'exportation des parties aériennes du maïs est sans effet sur les rendements mais l'introduction de l'engrais vert entraîne un gain de production significatif de 5,4 et $7,8 \%$. De même, l'interruption de la culture de maïs par la prairie temporaire exerce un effet bénéfique sur le maïs suivant qui a été évalué à 8,1 et $9,2 \%$ dans chacun des deux dispositifs.
\end{abstract}

maïs / interculture / prairie / résidus de culture / engrais verts

Summary - Long-term effect of a non-irrigated cropping system on grain yield of maize. Yields of non-irrigated maize crops were measured over a 21-yr period (1968-1988) in 1 experiment and over a 24-yr period (1968-1991) in another field located on the loamy sandy soils of southwest France. The experimental treatments studied were: i) continuous maize with aerial parts residues returned (TR); ii) continuous maize with aerial parts residues removed (TE); iii) continuous maize and a winter Italian rye-grass cover crop, sown in August and ploughed back in spring at the same time as the maize residue (RG); iv) rotation involving a continuous maize for 4 yr, followed by 3 yr temporary grassland (PT). In spite of the wide interannual variations due to fluctuations in seasonal water deficit, maize grain yields were not affected either by continuous maize aerial parts residues returned or removed. On the contrary, a trend toward a progressive increase in grain yield (0.76 and $1.19 \mathrm{q} / \mathrm{ha} / \mathrm{yr}$ respectively) was noted during the course of the experiment, probably due to genetic progress in plant breeding. The use of Italian rye-grass as a winter crop significantly enhanced grain production in the maize crops by 5.4 and $7.8 \%$ respectively. Likewise, a grain yield increase averaging 8.1 and $9.2 \%$ respectively was observed after the 3-yr fallow (grassland) period.

maize / annual cover crop / temporary grassland / crop residue / rye-grass

\section{INTRODUCTION}

Le maïs occupe annuellement près de 140000 ha dans le département des Landes où il représente l'essentiel des surfaces cultivées.

Depuis plusieurs décennies, la monoculture de cette espèce représente le système domi- nant. L'irrigation est généralisée dans la partie sableuse située au nord de la région, alors que la culture non irriguée est plus fréquente sur les limons sableux du sud.

L'aire d'extension et l'ancienneté de la culture continue du maïs dans la région peuvent conduire à poser le problème de la pérennité

\footnotetext{
${ }^{*}$ Correspondance et tirés à part
} 
d'un tel système dont on peut craindre a priori un effet négatif sur le potentiel de production du milieu.

De nombreux travaux, tels ceux de Snider (1956), Englehorn et al (1964), Barnhart et al (1978), Ketcheson (1980), Hooker et al (1983), Györffy (1984), Grove et Blevins (1988), ont pourtant montré que la culture continue du maïs pouvait être poursuivie pendant de longues périodes sans chute de rendement. II n'existe pas en France, à notre connaissance, d'étude de ce type portant sur une assez longue période (au moins 2 décennies), basée sur la comparaison entre les effets sur le rendement et la fertilité des sols d'un tel système et ceux produits par l'exportation systématique de résidus de culture, l'introduction d'un engrais vert en interculture ou encore l'interruption de la monoculture par une prairie temporaire.

C'est la raison pour laquelle 2 dispositifs ont été mis en place en 1967 dans la petite région agricole de Chalosse (département des Landes). Le choix de 2 dispositifs a été guidé par le souci de disposer en début d'expérience de parcelles possédant des comportements différents. L'objectif de l'étude était double :

- suivre l'évolution des rendements du maïsgrain en culture continue dans les conditions mêmes de la pratique agricole courante afin de mettre en évidence leur éventuelle baisse ou stagnation au cours du temps;

- comparer les statuts carbonés du sol selon les différents régimes de restitution humique (résidus de culture enfouis ou non, interruption de la monoculture par une interculture hivernale de ray-grass ou par une prairie temporaire de 3 ans).

Le choix des traitements a été guidé par le souci de vérifier diverses hypothèses, fréquemment avancées à l'époque de la mise en place de l'expérimentation, pour expliquer un éventuel effet négatif de la monoculture du maïs sur la fertilité à terme du milieu :

- l'exportation des parties aériennes végétatives avait pour objectif de vérifier l'hypothèse attribuant à l'accumulation d'acide para-coumarique produit lors de la décomposition des tiges de maïs (Hennequin et Juste, 1967) un rôle déterminant dans un possible phénomène de «fatigue du sol» ;

- l'introduction de la prairie temporaire et celle de l'engrais vert en période hivernale représentaient a priori un moyen de restaurer certaines caractéristiques de fertilité du sol liées à son statut organique et susceptibles d'être dégradées par la monoculture malgré la restitution d'une masse importante de résidus de récolte.

Les traitements mis en cuvre ont contribué aussi à fournir des éléments de réponse à des préoccupations nouvelles qui se sont fait jour pendant le déroulement de l'expérience : par exemple, la crise énergétique de 1973 a donné un poids particulier au traitement comportant l'exportation systématique des parties aériennes dont l'emploi, comme source énergétique (combustion directe ou méthanisation) a été un moment envisagé. De même, l'importance accordée actuellement au problème de pollution par les nitrates et la préconisation, relativement récente, d'intercultures d'engrais verts pour limiter cette dernière en jouant le rôle de "piège à nitrates", conduisent à examiner avec un intérêt accru le traitement où la monoculture est associée à un engrais vert en interculture.

Le but de ce travail est de présenter l'évolution des rendements en grain du maïs après 22 années d'expérimentation (1967 à 1988) dans un dispositif et après 25 années dans l'autre (1967 à 1991), ce bilan complétant celui déjà établi après les 10 premières années (Lubet et Juste, 1979).

\section{MATÉRIEL ET MÉTHODES}

\section{Dispositifs}

Les deux dispositifs, distants de $6 \mathrm{~km}$, sont situés à 20 $\mathrm{km}$ environ au sud de la ville de Mont-de-Marsan (lat $43,55 \mathrm{~N}$, long $0,30 \mathrm{O}$ ) sur le système géologique des sables fauves d'âge pliocène. Cette formation sabloargileuse forme un vaste manteau sur les collines de Chalosse. Elle est souvent recouverte par une faible épaisseur de dépôts limoneux plus ou moins remaniée.

Ces dépôts sont assez importants $(1,0$ à $1,20 \mathrm{~m})$ dans le secteur où est implanté le premier dispositif (dispositif de Serreslous). Ils ont donné naissance à un sol lessivé hydromorphe, à pourcentage élevé de limons (tableau I), passant sans transition en profondeur à un sable argileux compact. L'horizon de surface se caractérise par une forte tendance à la battance.

Le sol du deuxième dispositif (dispositif de Doazit) se caractérise par une épaisseur moindre et une texture sableuse plus grossière correspondant au recouvrement supérieur $(0,50 \mathrm{~m})$ qui repose sur un horizon concrétionné par des oxydes de fer et de manganèse. Cet horizon marque la discontinuité texturale avec une argile sableuse très compacte sous-jacente, limitant le 
Tableau I. Caractéristiques physico-chimiques des sols des 2 dispositifs en 1967.

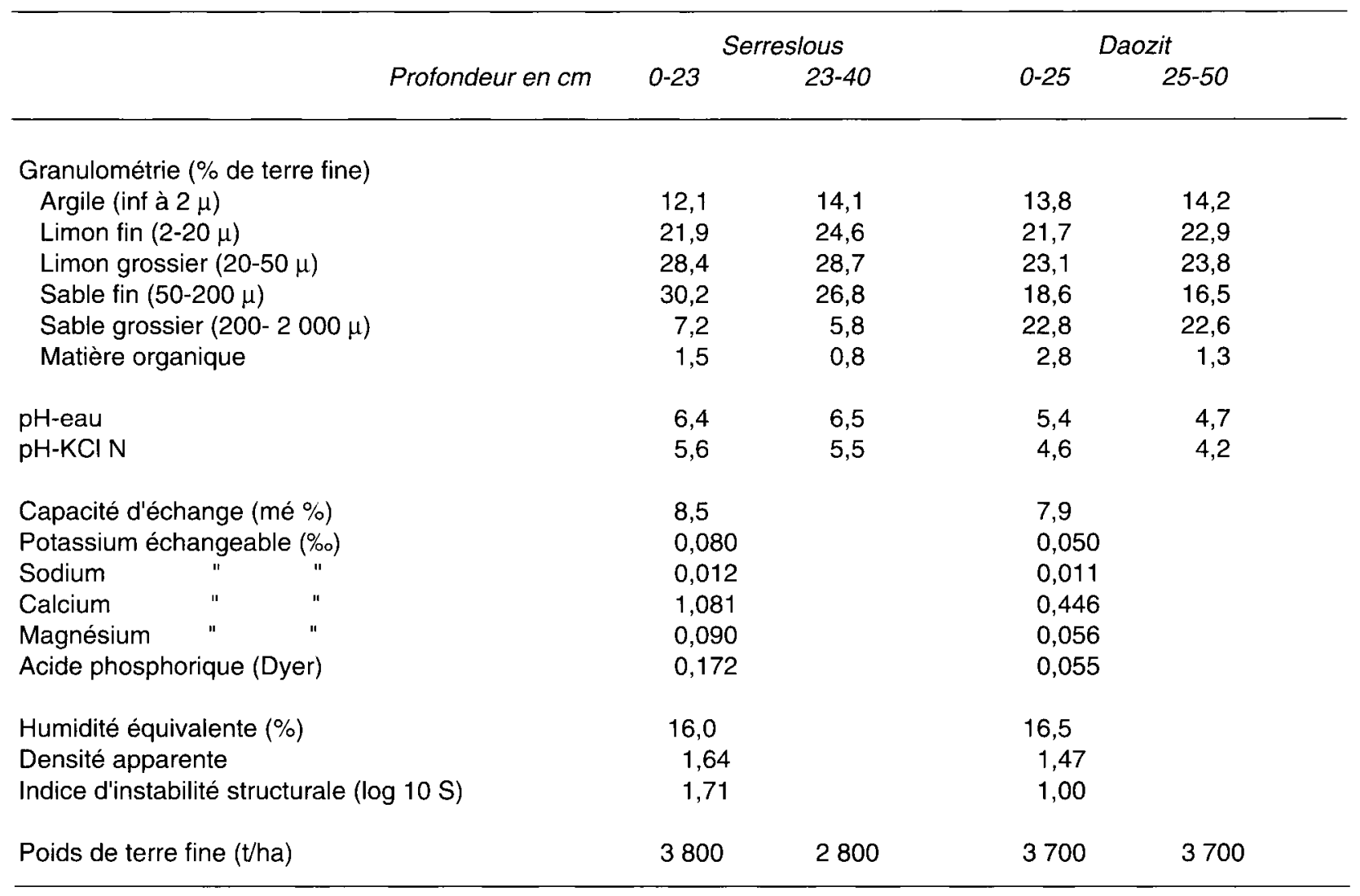

volume de sol exploitable par les racines; ce sol se caractérise par une plus grande richesse initiale en matière organique et une meilleure stabilité structurale que le précédent (tableau I).

Après son défrichement, la parcelle occupée par le dispositif de Doazit a été cultivée pendant plusieurs années par des successions triennales à base de blémaïs-trèfle incarnat avec jachère estivale, le maïs recevant régulièrement un abondant apport organique (50 t/ha de fumier de ferme). La parcelle occupée par le dispositif de Serreslous a été mise beaucoup plus anciennement en culture ; dans ce cas-là aussi la culture continue du maïs a été précédée par les mêmes successions blé-maïs-trèfle incarnat, mais avec des apports plus faibles d'amendements organiques.

\section{Protocole}

Chacun des 2 dispositifs de type bloc comporte les traitements suivants répétés 4 fois :

- i) traitement TR = monoculture de maïs dont la totalité des résidus de culture est incorporée au sol ;

- ii) traitement TE = monoculture de maïs dont la totalité des parties aériennes est exportée ;

- iii) traitement $R G$ = monoculture de maïs avec restitution des résidus de culture, suivie d'un engrais vert (ray-grass d'Italie) semé en août et enfoui au printemps avant le semis du maïs suivant ;

- iv) traitement PT = culture de maïs durant 4 années, avec restitution des résidus de culture, suivie d'une prairie temporaire de 3 ans. Au cours de l'expérience, 3 prairies ont ainsi été installées : 1968 à 1970, 1975 à 1977, 1982 à 1984.

La dimension des parcelles élémentaires est de $168 \mathrm{~m}^{2}(30 \times 5,6 \mathrm{~m})$ dans le dispositif de Doazit et de $190 \mathrm{~m}^{2}$ (34 x 5,6 m) dans celui de Serreslous.

Tous les travaux de préparation du sol sont réalisés dans les conditions même de la pratique agricole courante. Le labour, à l'occasion duquel sont enfouis les résidus de culture, est effectué fin mars-début avril, toujours à la même profondeur (contrôle par examen du profil). Des variétés demi-tardives, d'un usage courant dans la région, sont mises en œuvre lors du semis qui est fait à une densité moyenne de 75000 pieds/ha. La fertilisation phosphatée $(160 \mathrm{~kg} /$ ha $\left.\mathrm{P}_{2} \mathrm{O}_{5}\right)$ et potassique $\left(180 \mathrm{~kg} / \mathrm{ha} \mathrm{K}_{2} \mathrm{O}\right)$ est apportée en deux fois, partie avant labour et partie avant semis. La fertilisation azotée est fractionnée à raison de 30 unités avant labour, 30 unités avant semis et 140 unités en localisation dans l'interligne à $15 \mathrm{~cm}$ de profondeur au stade 8 feuilles. Le désherbage est assuré par la localisation de triazine dans la ligne de semis et par épandage en plein, en post-semis, d'un mélange de métolachlor (ou d'alachlore) et d'atrazine. 
Le semis de ray-grass d'Italie (variété tétraploïde alternative) est effectué manuellement courant août dans le maïs à raison de $25 \mathrm{~kg} / \mathrm{ha}$, les pluies de septembre assurant généralement une levée satisfaisante. Au cours des 10 premières années de l'expérience, un épandage de 30 unités d'azote a été occasionnellement réalisé dans l'ensemble du dispositif dans le courant de l'hiver en fonction du développement végétatif de l'engrais vert. Au moment de sa destruction par lacération au girobroyeur, le ray-grass mesure de 25 à $35 \mathrm{~cm}$ de hauteur et représente une biomasse totale de 2 à $5 \mathrm{t} / \mathrm{ha}$ de matière sèche totale suivant les années. Après sa destruction, l'engrais vert est abandonné en l'état à la surface du sol pendant les 2 semaines qui précèdent le labour.

La prairie temporaire est constituée d'un mélange de dactyle et de fétuque des prés, associée à du lotier et du trèfle blanc. Elle reçoit une fumure azotée fractionnée de $250 \mathrm{~kg} / \mathrm{ha}$ et est exploitée en fauche intégrale, l'ensemble des 3 ou 4 coupes annuelles représentant une production de matière sèche qui varie de 12 à $18 \mathrm{t} /$ ha. Le retournement est effectué un mois environ avant le semis du maïs et une fumure de redressement potassique $\left(300 \mathrm{~kg} / \mathrm{ha} \mathrm{K}_{2} \mathrm{O}\right)$ est incorporée à cette occasion.

\section{Données météorologiques}

Les données climatiques proviennent des relevés de Météo-France réalisés à la station de Montaut (40), située à $3 \mathrm{~km}$ du dispositif Doazit et à $8 \mathrm{~km}$ de celui de Serreslous pour la période 1973-1991 (température et pluviométrie). Les données concernant I'ETP (Penman) sont issues de la station de Mont-de-Marsan (40) qui est située à $25 \mathrm{~km}$ des dispositifs. Les données concernant la pluviométrie pour la période 19681972 proviennent également de cette station sachant que la pluviométrie cumulée de juin-juillet-août est de 20 à $30 \mathrm{~mm}$ inférieure à celle de la même période à la station de Montaut.

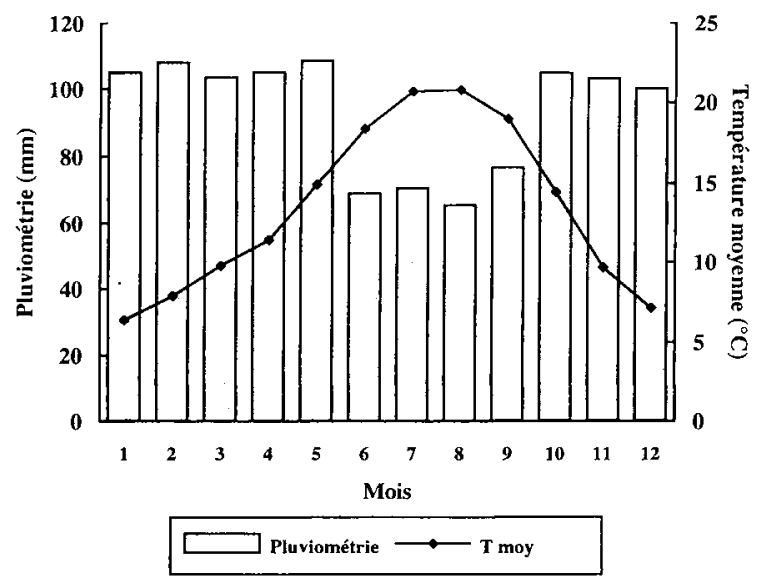

Fig 1. Caractéristiques climatiques moyennes des sites expérimentaux (période 1973-1991). Données de la station météorologique de Montaut.
La pluviométrie annuelle moyenne des sites au cours de la période 1973 à 1991 a été de $1120 \mathrm{~mm}$ avec des minima situés entre juin et septembre (fig 1). Pour la période considérée, I'ETP moyenne a été de $783 \mathrm{~mm}$, la moyenne des températures minimales $8,3^{\circ} \mathrm{C}$ et la moyenne des températures maximales $18,5^{\circ} \mathrm{C}$.

\section{Détermination des rendements du maïs}

Les rendements ont été contrôlés de 1968 à 1988 (21 années) dans le dispositif Serreslous et de 1968 à 1991 (24 années) dans le dispositif Doazit. Ils sont estimés par pesée de la récolte manuelle des 2 rangs centraux de chaque parcelle.

\section{Analyse statistique des résultats}

Les moyennes des rendements de chaque traitement ont fait l'objet d'une analyse de variance année par année. Une analyse pluriannuelle portant sur 21 ans dans le dispositif Serreslous et sur 24 ans dans celui de Doazit a également été faite en fin d'expérience en vue de comparer les effets moyens des traitements $T R$, TE, et RG. L'effet moyen du traitement prairie sur la production du maîs suivant a fait l'objet d'une deuxième analyse de variance pluriannuelle intégrant les 12 et 15 années au cours desquelles le maïs était effectivement présent sur les parcelles correspondant à ce traitement.

Pour tenter d'expliquer les variations du rendement attribuables aux paramètres climatiques et/ou au temps, on a fait appel à un calcul de régressions linéaires multiples. Dans le cas des régressions progressives, la probabilité d'introduction des différentes variables a été fixée à $10 \%$.

\section{RÉSULTATS}

Les tableaux II et III présentent l'ensemble des rendements mesurés année par année pour l'ensemble des traitements ainsi que l'interprétation statistique de l'analyse de variance.

\section{Système à base de culture continue de maïs avec restitution des résidus de culture (Traitement TR)}

Les rendements moyens calculés pour l'ensemble de la période expérimentale sont du même ordre de grandeur pour les 2 dispositifs (76,6 et 74,5 q/ha à Serreslous et Doazit, respectivement). 
Tableau II. Rendement en grain du maïs : dispositif de Serreslous.

\begin{tabular}{|c|c|c|c|c|c|}
\hline \multirow{2}{*}{ Années } & \multicolumn{4}{|c|}{ Rendements ( $q / h a)$} & \multirow{2}{*}{ Contraste } \\
\hline & $T R$ & $T E$ & $R G$ & $P T$ & \\
\hline 1968 & 83,00 & 81,13 & 79,13 & & NS \\
\hline 1969 & $60,60^{a}$ & $59,90^{a}$ & $53,60^{b}$ & & * \\
\hline 1970 & 66,10 & 71,13 & 65,10 & & NS \\
\hline 1971 & $82,53^{b}$ & $83,52 \mathrm{~b}$ & $91,30^{a}$ & $93,50^{a}$ & * \\
\hline 1972 & $60,55^{b}$ & $61,10^{b}$ & $59,38^{b}$ & 68,38 a & NS \\
\hline 1973 & $88,20^{a}$ & $85,13^{b}$ & 89,55 a & $88,65^{a}$ & * \\
\hline 1974 & 82,32 & 82,13 & 87,63 & 86,45 & * \\
\hline 1975 & $46,33 b$ & $43,90^{b}$ & $59,00^{a}$ & & * \\
\hline 1976 & $62,10^{b}$ & $64,75^{b}$ & $74,78^{a}$ & & * \\
\hline 1977 & 64,57 & 63,80 & 64,20 & & NS \\
\hline 1978 & $80,85 a b$ & $79,85^{b}$ & $86,63^{a}$ & $81,53 a b$ & $\star$ \\
\hline 1979 & 81,25 & 84,72 & 85,15 & 83,20 & NS \\
\hline 1980 & 81,52 & 78,70 & 79,02 & 84,98 & NS \\
\hline 1981 & $84,43^{b}$ & $82,68^{b}$ & $94,88^{a}$ & $94,20^{a}$ & * \\
\hline 1982 & $71,90 \mathrm{~b}$ & $69,18^{b}$ & $77,97^{a}$ & & * \\
\hline 1983 & $79,65^{b}$ & $80,82^{b}$ & $87,63^{a}$ & & * \\
\hline 1984 & 73,30 & 74,55 & 78,60 & & NS \\
\hline 1985 & $81,28^{b}$ & $82,32 b$ & 91,65 a & $93,30^{a}$ & * \\
\hline 1986 & $65,82 \mathrm{bc}$ & $60,38^{c}$ & 71,68 & $80,45^{a}$ & * \\
\hline 1987 & 119,72 & 120,60 & 120,38 & 124,60 & NS \\
\hline 1988 & $93,00^{b}$ & $91,90^{b}$ & $99,18^{a}$ & $100,00^{a}$ & * \\
\hline Moyenne/21 ans & $76,62^{b}$ & $76,29 \mathrm{~b}$ & $80,78 a$ & & * \\
\hline (A) & $83,46^{c}$ & $82,75^{c}$ & $88,03^{b}$ & 90,19 a & * \\
\hline
\end{tabular}

Sur une même ligne, les valeurs suivies par une même lettre ne sont pas significativement différentes (test de Newman-Keuls au seuil de $5 \%$ ). Contraste : TE + TR/RG ou TE + TR/RG + PT suivant les années ; NS : non significatif ; ${ }^{*}$ : probabilité $<5 \%$; A : moyenne des rendements obtenus les années suivant le retournement des prairies ; TR : monoculture de maïs, parties aériennes restituées ; TE : monocultures de maïs, parties aériennes exportées ; RG : monoculture de maïs + interculture de ray-grass ; PT : culture de maïs (4 ans) + prairie temporaire ( 3 ans).

Corroborant une observation antérieure, (Lubet et Juste, 1979), on constate de fortes variations interannuelles de rendement (figs 2 et 3 ) du même ordre de grandeur dans les 2 dispositifs comme le démontre la similitude des variances $(228,6$ et 232,0$)$.

Comme on pouvait facilement le prévoir, une première analyse des données climatiques fait apparaître que l'origine de ces variations interannuelles du rendement est due principalement au déficit du bilan hydrique estival. En effet, si l'on se réfère au tableau IV dans lequel figurent les bilans hydriques climatiques $(\mathrm{BHC}=\mathrm{P}-\mathrm{ETP})$ mensuels des différentes années ainsi que les valeurs cumulées de cet indice pour la période de juin à août (fig 4), on constate que ce bilan est systématiquement déficitaire $(-4$ à $-263 \mathrm{~mm})$ pour cette période. Seule l'année 1977, extrêmement humide, fait exception à cette règle en raison de la pénalisation des rendements par un printemps très pluvieux qui a conduit à retarder anormalement la date des semis. Dans ce qui suit, les données relatives à cette année ne seront donc pas prises en compte.

L'estimation, par une régression linéaire multiple, de l'influence sur le rendement de la variable année (durée de l'expérience) et des variables (P-ETP) mensuelles d'avril à septembre montre qu'elles expliquent à elles seules 70,2 (Serreslous) et $58,2 \%$ (Doazit) de la variation des rendements. La part d'explication respective attribuable aux variables ( $P-E T P)$ et année a été estimée pour chacun des dispositifs en utilisant une méthode de régression linéaire pro- 
Tableau III. Rendement en grain du maïs : dispositif de Doazit.

\begin{tabular}{|c|c|c|c|c|c|}
\hline \multirow[t]{2}{*}{ Années } & \multirow[b]{2}{*}{$T R$} & \multicolumn{2}{|c|}{ Rendements ( $q / h a)$} & \multirow[b]{2}{*}{$P T$} & \multirow[t]{2}{*}{ Contraste } \\
\hline & & $T E$ & $R G$ & & \\
\hline 1968 & 59,40 & 60,10 & 62,50 & & NS \\
\hline 1969 & 78,52 & 77,80 & 77,90 & & NS \\
\hline 1970 & 76,50 & 77,00 & 79,90 & & NS \\
\hline 1971 & $75,07 b$ & $78,72^{b}$ & $77,13^{b}$ & 85,43 a & * \\
\hline 1972 & 82,53 & 81,68 & 86,13 & 86,13 & NS \\
\hline 1973 & 91,50 & 92,05 & 94,20 & 91,65 & NS \\
\hline 1974 & $86,30^{b}$ & $85,95 b$ & 92,15 ab & 94,05 & * \\
\hline 1975 & 52,67 & 55,50 & 64,90 & & * \\
\hline 1976 & 63,90 & 66,00 & 70,30 & & NS \\
\hline 1977 & 78,75 & 78,57 & 80,05 & & NS \\
\hline 1978 & $87,03^{b}$ & $91,30^{a}$ & 92,12 a & $93,20^{a}$ & * \\
\hline 1979 & $93,30^{b}$ & $90,72 b$ & $100,18^{a}$ & $101,15^{b}$ & * \\
\hline 1980 & $73,72^{b}$ & $73,58^{b}$ & 85,07 a & $84,30^{a}$ & * \\
\hline 1981 & 82,43 & 82,30 & 85,97 & 83,53 & NS \\
\hline 1982 & 88,38 & 87,40 & $94,55 \mathrm{a}$ & & * \\
\hline 1983 & 92,57 a & $88,30^{b}$ & $94,50^{a}$ & & * \\
\hline 1984 & $44,50^{b}$ & $44,97 b$ & $53,63^{a}$ & & * \\
\hline 1985 & $67,57^{b}$ & $68,72^{b}$ & 78,32 a & $81,55^{a}$ & * \\
\hline 1986 & $58,30^{b}$ & $57,23^{b}$ & $61,88 a b$ & $66,90^{a}$ & * \\
\hline 1987 & 97,70 & 99,45 & 100,77 & 104,45 & * \\
\hline 1988 & 82,78 & 82,78 & 90,35 & 90,05 & * \\
\hline 1989 & $64,86^{b}$ & $65,04 b$ & $76,40^{a}$ & 72,39 a & * \\
\hline 1990 & $44,15^{b}$ & $42,34 \mathrm{~b}$ & $51,11 a b$ & 53,98 a & * \\
\hline 1991 & $66,51^{b}$ & $66,28^{b}$ & $77,62^{a}$ & $70,74^{b}$ & * \\
\hline Moyenne/24 ans & $74,54 \mathrm{~b}$ & $74,74 b$ & $80,32^{a}$ & & * \\
\hline (A) & $76,92^{b}$ & $77,21^{b}$ & $83,29 a$ & $83,97^{a}$ & * \\
\hline
\end{tabular}

Sur une même ligne, les valeurs suivies par une même lettre ne sont pas significativement différentes (test de Newman-Keuls au seuil de $5 \%$ ). Contraste : TE + TR/RG ou TE + TR/RG + PT suivant les années ; NS : non significatif ; ${ }^{*}$ : probabilité $<5 \%$; A : Moyenne des rendements obtenus les années suivant le retournement des prairies; TR : monoculture de maïs, parties aériennes restituées; TE : monocultures de maïs, parties aériennes exportées ; RG : monoculture de maïs + interculture de ray-grass ; PT : culture de maïs ( 4 ans) + prairie temporaire ( 3 ans).

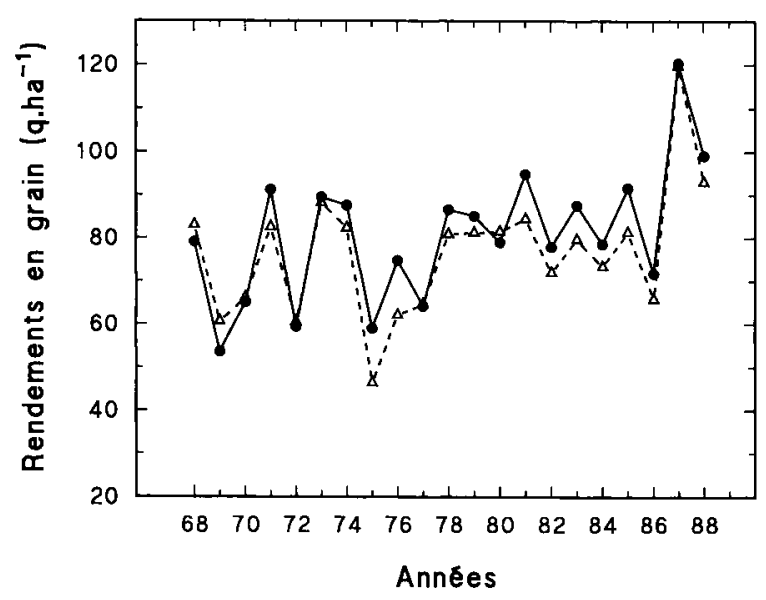

Fig 2. Influence de l'année et de l'interculture d'engrais vert sur le rendement en grain du maïs (dispositif de Serreslous). $\nabla-\cdots \nabla:$ monoculture de maìs, tiges restituées (TR) ; $\bullet$ : monoculture de maïs, tiges restituées + interculture de raygrass (RG).

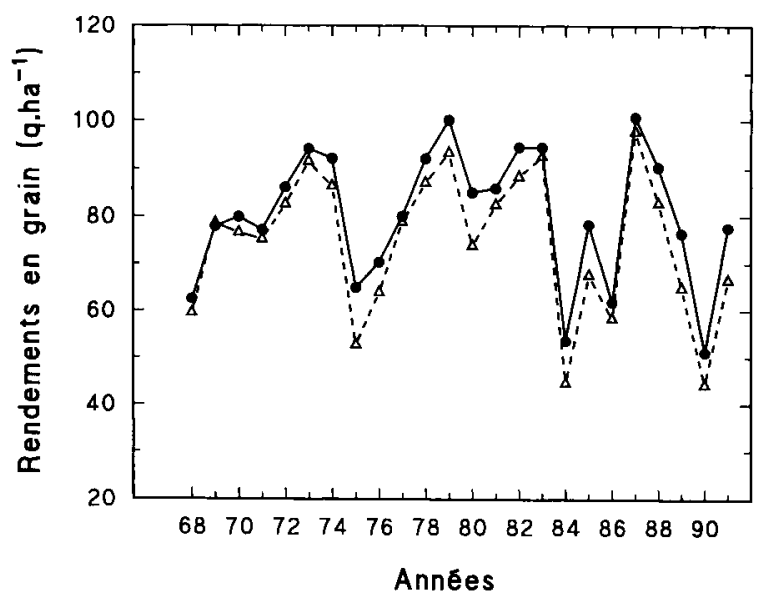

Fig 3. Influence de l'année et de l'interculture d'engrais vert sur le rendement en grain du maïs (dispositif de Doazit). $\nabla---\nabla$ : monoculture de maïs, tiges restituées $(T R) ; \bullet \bullet$ : monoculture de maïs, tiges restituées + interculture de raygrass (RG). 
Tableau IV. Bilan hydrique climatique (P-ETP) mensuel de 1968 à 1991.

\begin{tabular}{|c|c|c|c|c|c|c|}
\hline \multirow[t]{2}{*}{ Années } & \multicolumn{6}{|c|}{ Bilan hydrique : $P$ - ETP (en $\mathrm{mm})$} \\
\hline & Avril & $M a i$ & Juin & Juillet & Août & Septembre \\
\hline 1968 & -10 & -7 & -73 & -99 & 31 & 29 \\
\hline 1969 & 46 & -30 & -40 & -145 & -29 & 121 \\
\hline 1970 & 5 & -68 & -18 & -127 & -32 & -70 \\
\hline 1971 & 42 & 34 & 6 & -45 & -39 & -3 \\
\hline 1972 & 9 & -30 & -38 & -115 & 42 & -24 \\
\hline 1973 & -31 & -28 & -53 & 41 & -89 & 27 \\
\hline 1974 & -10 & 8 & -81 & -76 & -70 & 81 \\
\hline 1975 & 23 & 62 & -46 & -138 & -78 & 59 \\
\hline 1976 & 11 & -68 & -140 & -79 & 10 & 45 \\
\hline 1977 & -5 & 130 & 50 & -5 & 66 & -70 \\
\hline 1978 & 94 & -1 & 8 & -64 & -79 & -5 \\
\hline 1979 & 101 & 61 & -39 & -65 & -48 & -18 \\
\hline 1980 & -52 & 148 & -23 & -55 & -64 & -42 \\
\hline 1981 & 30 & 60 & -89 & -34 & -57 & 56 \\
\hline 1982 & -92 & -56 & -45 & -68 & -14 & -10 \\
\hline 1983 & 52 & -14 & -108 & -35 & 19 & -39 \\
\hline 1984 & -74 & 41 & -43 & -146 & -73 & 49 \\
\hline 1985 & -30 & 31 & -63 & -62 & -78 & -97 \\
\hline 1986 & 140 & -11 & -92 & -129 & -42 & -24 \\
\hline 1987 & 10 & -81 & 13 & 14 & -31 & -47 \\
\hline 1988 & 92 & -4 & -57 & -91 & -72 & -18 \\
\hline 1989 & 223 & -82 & -99 & -67 & -95 & -41 \\
\hline 1990 & 23 & -46 & -1 & -128 & -82 & -53 \\
\hline 1991 & 37 & -5 & -47 & -46 & -107 & 61 \\
\hline
\end{tabular}

gressive excluant l'introduction des variables dès que la probabilité de rejet de ces dernières dans le modèle devient supérieure à 10\% (tableau V). On obtient ainsi les régressions suivantes :

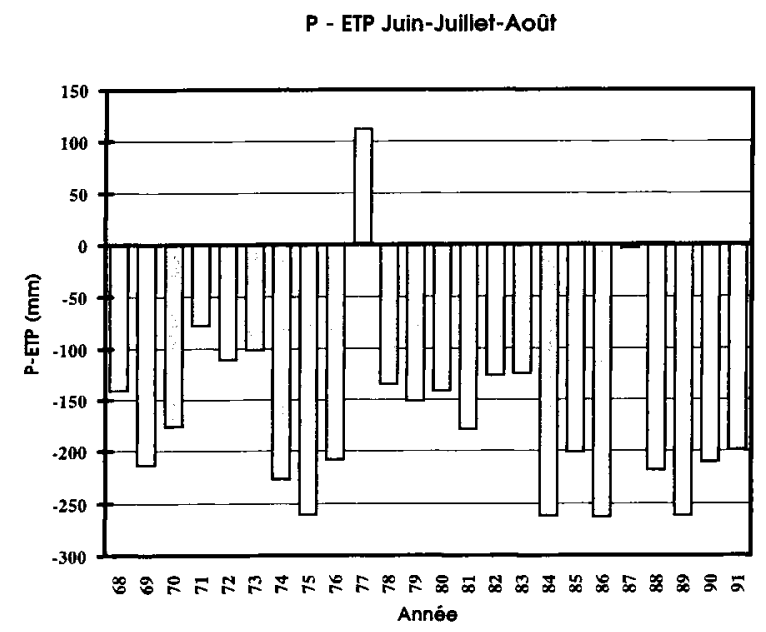

Fig 4. Bilan hydrique climatique (P-ETP) cumulé sur les mois de juin, juillet et août. Période 1968-1991. Données des stations météorologiques de Montaut (pluviométrie de 1973 à 1991) et de Mont-de-Marsan (pluviométrie de 1968 à 1972 et ETP).
- Dispositif de Serreslous : Rendement $(q / \mathrm{ha})=$ 0,759 (année) $+0,102$ (P-ETP de juin) $+0,194$ (P-ETP de juillet) $+89,55$.

Le calcul de régression progressive conduit donc à un modèle dans lequel ne figurent que la variable année et les bilans hydriques climatiques (P-ETP) relatifs uniquement aux mois de juin et de juillet. Ce résultat confirme donc l'importance de la pluviosité estivale sur le déterminisme du rendement. II met en évidence une tendance à l'accroissement des rendements avec le temps que l'on peut évaluer à 0,76 q/ha/an.

- Dispositif de Doazit : Rendement ( $q / h a)=$ 0,218 (P-ETP de juillet) $+0,113$ (P-ETP d'août) $+96,33$

Contrairement à ce qui a été observé dans le dispositif Serreslous, il n'est donc pas possible, en procédant ainsi, de mettre en évidence dans ce cas un effet année, seuls les bilans hydriques climatiques des mois de juillet et d'août apparaissant comme les déterminants majeurs du rendement. 
Tableau V. Résultats des régressions linéaires multiples progressives entre le rendement et les variables : année, bilans hydriques mensuels (P-ETP).

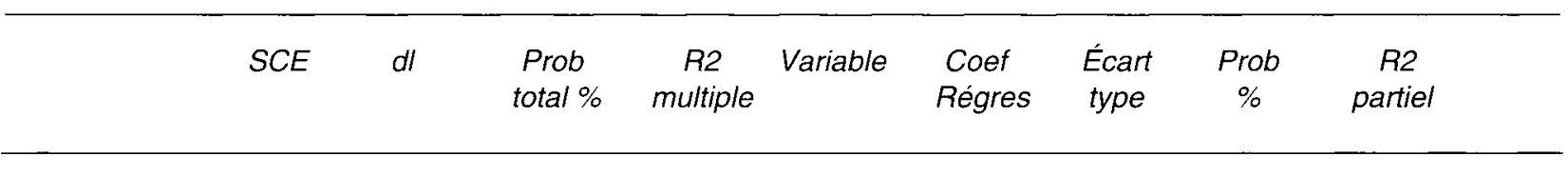

Serreslous 20 ans

\begin{tabular}{|c|c|c|c|c|c|c|c|c|c|}
\hline Totale & 4420 & 19 & & & Année & 0,759 & 0,343 & 4,3 & 0,228 \\
\hline Régression & 3001 & 3 & 0,04 & 0,679 & Juin & 0,102 & 0,056 & 8,4 & 0,17 \\
\hline Résiduelle & 1419 & 16 & & & Juillet & 0,194 & 0,044 & 0,05 & 0,548 \\
\hline
\end{tabular}

Doazit 23 ans

$\begin{array}{lrrlllllll}\text { Totale } & 5318 & 22 & & & & & & & \\ \text { Régression } & 2660 & 2 & 0,10 & 0,500 & \text { Juillet } & 0,218 & 0,050 & 0,04 & 0,477 \\ \text { Résiduelle } & 2658 & 20 & & & \text { Août } & 0,113 & 0,060 & 7,10 & 0,151\end{array}$

Doazit 11 ans (répartis entre 1968 et 1986)

\begin{tabular}{|c|c|c|c|c|c|c|c|c|c|}
\hline Totale & 1054 & 10 & & & Année & 1,190 & 0,490 & 3,98 & 0,387 \\
\hline Régression & 407 & 1 & 3,98 & 0,387 & & & & & \\
\hline Résiduelle & 647 & 9 & & & & & & & \\
\hline
\end{tabular}

SCE : somme des carrés des écarts ; dl : degrés de liberté ; Prob : probabilité en \%.

Cette différence peut s'expliquer par les caractéristiques particulières du sol du dispositif Doazit qui rendent le couvert végétal plus sensible au stress hydrique, mais aussi par la prolongation de l'expérience jusqu'en 1991, date qui clôture une série de 3 années à déficit hydrique estival très important (fig 4). Dans ces conditions, l'effet des variables climatiques devient prépondérant et pourrait ainsi masquer un éventuel effet du temps. Pour vérifier cette hypothèse, on a établi une régression progressive liant (P-ETP) mensuel et rendement en ne retenant que les 11

Tableau VI. Bilan hydrique des mois de janvier à mai. Moyenne de la période 1973-1991.

\begin{tabular}{lccr}
\hline Mois & $P(\mathrm{~mm})$ & ETP $(\mathrm{mm})$ & $P-E T P$ \\
& & & \\
\hline Janvier & 105,0 & 11,2 & 93,8 \\
Février & 108,1 & 22,8 & 85,3 \\
Mars & 103,5 & 50,8 & 52,7 \\
Avril & 105,1 & 76,6 & 28,5 \\
Mai & 108,6 & 101,0 & 7,6 \\
Cumul & 530,3 & 262,4 & $+267,9$ \\
& & & \\
\hline
\end{tabular}

années de la période 1968-1983 pour lesquelles le bilan hydrique climatique cumulé des mois de juin à août est compris entre -80 et $-180 \mathrm{~mm}$. C'est dans cet intervalle que se situent en effet les valeurs les plus probables du bilan hydrique cumulé de ces 3 mois pour la période considérée. En procédant de la sorte on homogénéise, au moins en partie, l'effet du climat. La régression progressive (tableau $V$ ) met alors en évidence un accroissement des rendements uniquement lié au temps, de la forme:

Rendement $(q / h a)=1,19$ (année $)+72,43$

\section{Autres système de culture}

\section{Exportation des résidus de récolte}

Confirmant les résultats des 11 premières années de l'expérimentation (Lubet et Juste, 1979), il apparaît clairement que la non-restitution au sol des tiges, feuilles et spathes (traitement TE) ne modifie absolument pas le rendement en grain. Pour l'ensemble de la période expérimentale, la moyenne générale du traitement TE est strictement identique à celle de TR dans chacun des deux dispositifs. 


\section{Introductrion de l'interculture de ray-grass}

L'introduction systématique d'un ray-grass durant la période hivernale et son enfouissement avant la mise en place du maïs suivant (traitement RG) conduisent à un accroissement moyen des rendements, qui, pour la totalité de la durée de l'essai et par comparasion avec le traitement TR, a été évalué respectivement à $5,4 \%(4,2 \mathrm{q} / \mathrm{ha})$ dans le dispositif Serreslous et à $7,8 \%(5,8 \mathrm{~g} / \mathrm{ha})$ dans le dispositif Doazit.

Lors des 2 premières années de l'expérience l'introduction de l'engrais vert affecte négativement la production en grain dans le dispositif de Serreslous (chute significative de $7 \mathrm{q} / \mathrm{ha}$ en 1969). Un tel effet dépressif initial n'a pas été observé dans le dispositif de Doazit.

L'expérience montre également le caractère croissant de l'amélioration de la production de grain provoquée par l'introduction de l'engrais vert : en effet, lors des 10 premières années, l'augmentation moyenne des rendements pour ce traitement est de $2,7 \mathrm{q} / \mathrm{ha}(3,9 \%)$ et $4,0 \mathrm{q} / \mathrm{ha}$ $(5,5 \%)$ à Serreslous et Doazit respectivement, alors que, pour la période 1978 à 1988, elle atteint $5,5 \mathrm{q} / \mathrm{ha}(6,0 \%)$ et $6,3 \mathrm{q} / \mathrm{ha}(8,0 \%)$. Dans le cas particulier de l'expérience de Doazit qui a été poursuivie jusqu'en 1991, année clôturant une série de 3 années à fort déficit hydrique estival, l'effet améliorateur du ray-grass est encore beaucoup plus marqué : $7,2 \mathrm{q} /$ ha $(10,2 \%)$ pour la période allant de 1982 à 1991.

\section{Introduction d'une prairie temporaire}

Une analyse statistique pluriannuelle des rendements, réalisée les années pour lesquelles le maïs est présent dans l'ensemble des traitements, met en évidence une action bénéfique du précédent prairie sur la production de grain. Pour la durée complète de l'expérience, cet effet a été estimé à $8,1 \%$ dans le dispositif de Serreslous et à $9,2 \%$ dans celui de Doazit.

Contrairement à la tendance qui s'était dégagée au cours du premier cyle de l'expérience (Lubet et Juste, 1979), on ne note pas, au cours des 4 années de culture de maïs suivant la prairie, une décroissance de l'arrière-effet de cette dernière au cours du temps.

\section{DISCUSSION}

\section{Évolution des rendements en culture continue de maïs avec restitution des résidus de récolte}

Cette étude souligne en premier lieu le rôle déterminant du régime hydrique estival et plus précisément celui du bilan hydrique climatique du mois de juillet sur le déterminisme du rendement en grain des monocultures non irriguées du maïs dans le secteur considéré. Le décalage dans le temps des réponses culturales au déficit climatique (mois de juin et juillet à Serreslous, mois de juillet et d'août à Doazit) s'explique par des dynamiques de dessèchement du sol et d'installation du système racinaire qui sont très différentes dans les deux situations (Arrouays et al, 1989). Le sol limoneux hydromorphe à Serreslous se caractérise en effet par une plus grande sensibilité à la sécheresse de juin liée à une mauvaise exploitation de la ressource en eau par les racines, alors que, dans le sol sablolimoneux de Doazit, la culture est surtout pénalisée par la faiblesse de la réserve hydrique en fin de campagne. Ce sont ces fluctuations climatiques intervenant au cours de ces périodes clés qui expliquent la grande variabilité interannuelle des rendements observée au cours de l'expérience.

Malgré cette grande variabilité, il apparaît que la monoculture ne s'accompagne pas d'une baisse des rendements qui aurait pu traduire une détérioration, au cours du temps, du potentiel de production du sol imputable à ce mode d'exploitation.

Ce résultat est en convergence avec la synthèse réalisée par Englehorn et al (1964), après analyse de l'ensemble des essais de longue durée comportant des monocultures de maïs réalisés durant la première moitié de ce siècle aux USA, notamment dans les États du Minnesota, de l'Ohio et de l'lllinois. Ces auteurs ont également montré qu'un seul apport de $140 \mathrm{~kg}$ d'azote/ha, effectué après 38 années de monoculture, était suffisant pour masquer l'effet de tous les précédents expérimentés et plus particulièrement de celui constitué par des Légumineuses qui était considéré jusqu'alors comme 
indispensable à la poursuite de culture continue. De nombreuses autres expérimentations sont venues par la suite corroborer ce premier résultat fondamental, notamment celles citées par Barnhart et al (1978) dans l'lowa (19 ans), Hooker et al (1983) dans le Kansas (14 et 21 ans), Györffy (1984) en Allemagne (22 ans), Grove et Blevins (1988) dans le Kentucky (16 ans).

Le grand nombre de données accumulées au cours de la présente étude a permis de dissocier l'estimation de l'effet du climat de celui de la durée de l'expérience sur la production du grain. On peut mettre ainsi en évidence un accroissement moyen annuel du rendement en grain, uniquement corrélé au temps, de 0,76 et $1,19 \mathrm{q} / \mathrm{ha} /$ an respectivement à Serreslous et Doazit. Cet accroissement, très vraisemblablement attribuable à l'amélioration génétique du matériel végétal au cours du temps, est de même ordre de grandeur que celui cité par celui cité par Ketcheson (1980) pour l'Ontario (1,14 q/ha/an de 1957 à 1977) et par Derieux et al (1987) en France (0,8 q/ha/an pour la période de 1950 à 1980).

\section{Effet des différents régimes de restitution organique sur l'évolution des rendements}

L'absence d'effet de l'enlèvement des résidus de culture sur le rendement des cultures suivantes a sans doute pour origine un faible impact de cette pratique sur les caractéristiques du sol, la fertilisation appliquée tenant largement compte des exportations. L'absence d'effet de l'exportation maximale de biomasse sur la production du maïs-grain, phénomène déjà constaté depuis longtemps par Page et Willard (1946) et rappelé ultérieurement par Dutil et al (1982) pour l'ensemble des cultures céréalières, montre qu'une utilisation éventuelle des partie aériennes du maïs à d'autres fins que le maintien de la fertilité du sol, définie uniquement en termes de rendement, est envisageable. Par ailleurs, l'hypothèse selon laquelle des toxines produites lors de la décomposition dans le sol des tiges de maïs exerceraient une action dépressive sur la culture suivante (Hennequin et Juste, 1967), semble devoir être écartée.

L'interprétation statistique pluriannuelle illustre bien, par contre, l'intérêt que peut présenter, dans la région considérée, l'interculture d'engrais vert ou l'introduction d'une prairie temporaire pour les cultures suivantes de maïs. On note cependant que l'influence bénéfique de l'intercul- ture ne ressort clairement qu'après analyse des deux décennies de données. De fortes variations dans l'intensité et la nature de l'effet de l'engrais vert sont en effet enregistrées d'une année sur l'autre. Ces variations sont dues pour partie à la qualité de l'enfouissement mais aussi aux caractéristiques climatiques de l'année. Les années à fort déficit hydrique estival sont dans l'ensemble celles où l'action bénéfique de l'interculture est la plus marquée. Cette variabilité, liée aux fluctuations climatiques interannuelles, est vraisemblablement à l'origine des résultats contradictoires obtenus par de nombreux auteurs (Giddens et al, 1965 ; Monnier, 1965 ; Scott et al, 1987 ; Wagger et Mengel, 1987 ; Wagger, 1989a, b ; Martinez et Guiraud, 1990) qui ont attribué d'éventuels effets négatifs principalement à l'augmentation du déficit hydrique provoquée par le passage de l'interculture. Le tableau VI montre qu'une telle hypothèse est difficilement recevable dans le contexte climatique du Sud-Ouest atlantique français, caractérisé par un bilan hydrique largement exédentaire en fin d'hiver et au printemps.

L'influence bénéfique exercée par la prairie et l'inter-culture de ray-grass ne semble pas, a prio$r i$, pouvoir s'expliquer en termes d'amélioration du statut carboné du sol puisque la non restitution des résidus de culture (TE), qui représentent pourtant une masse importante de carbone, n'entraîne pas, par rapport au traitement dans lequel ils sont restitués (TR), une diminution du rendement en grain de maïs. L'origine de cet effet bénéfique semble plutôt à rechercher dans une possible amélioration des caractéristiques de comportement du sol due à l'effet structurant du système racinaire des intercultures, ou, plus vraisemblablement, à l'induction d'une modification de la dynamique de l'azote du sol mieux adaptée aux besoins du maïs, notamment en année sèche.

\section{CONCLUSION}

Parmi les monocultures traditionnelles qui caractérisent le Sud-Ouest atlantique français comme celles de la vigne, de la forêt, des arbres fruitiers et du maïs, c'est indiscutablement cette dernière qui suscite sporadiquement le plus de craintes quant à ses effets négatifs éventuels sur les potentialités actuelles de production du milieu. Dans la mesure où l'on considère que le maintien, voire l'accroissement au cours du temps 
des rendements, vérifié au cours des 2 dernières décennies, préfigure l'évolution future, le présent travail démontre que ces craintes ne sont pas a priori fondées.

Des modifications sensibles du régime des restitutions organiques telles que celles consistant en l'introduction d'une interculture hivernale ou d'une prairie temporaire sont de nature à améliorer sensiblement à terme le protentiel de production des sols soumis à la monoculture du maïs. II est cependant évident que les contraintes techniques et surtout économiques (changement du système de production dans le cas de l'introduction de la prairie par exemple) qu'impliquent de telles modifications en limitent l'éventuelle généralisation.

\section{REMERCIEMENTS}

Les auteurs expriment leur vive reconnaissance aux 2 agriculteurs, MM Demen et Laffargue, qui ont bien voulu accepter la mise en place de dispositifs de longue durée sur leurs exploitations.

\section{RÉFÉRENCES}

Arrouays D, Chossat JC, Darthout R, Nicoullaud B, Houdusse M (1989) Dessèchement de trois types de sol des Landes sous maïs-grain. Application à la gestion de l'eau. Bull GFHN 26, 31-50

Barnhart SL, Shrader WD, Webb Jr (1978) Comparison of soil properties under continuous corn grain and silage cropping systems. Agron J 70, 835-837

Derieux M, Darrigrand M, Gallais A, Barriere Y, Bloc D, Montalant $Y$ (1987) Estimation du progrès génétique réalisé chez le maïs grain en France entre 1950 et 1985. agronomie 7, 1-11

Dutil P, Juste C, Monnier G, Remy JC (1982) Paille et fertilité des sols. Point sur les travaux réalisés en France. INRA-Service public, $60 \mathrm{p}$

Englehorn AJ, Pesek J, Shrader WD (1964) Role of nitrogen, phosphorus and potassium fertilizers in continuous corn culture on Nicollet and Webster soils. Res Bull 522, 21 p, Agric Home Econ Exptl Stat, lowa State Univ Sci Technol

Giddens J, Arshad S, Rogers TH (1965) Effect of nitrogen and green manures on corn yield and properties of a Cecil soil. Agron J 57, 466-469
Grove JH, Blevins RL (1988) Correcting soil acidification in continuous corn (Zea mays $\mathrm{L}$ ): $\mathrm{N}$ rates, tillage and time. Commun Soil Sci Plant Anal 19, 1331-1342

Györffy B (1984) Fruchtfolge und monokultur in Maisanbau. Tag Ber Akad Landwirtsch Wiss DDR 224,119-123

Hennequin JR, Juste C (1967) Présence d'acides phénols libres dans le sol. Étude de leur influence sur la germination et la croissance des végétaux. Ann Agron 18, 545-569

Hooker ML, Gwin RE, Herron GM, Gallagher $P$ (1983) Effects of long-term, annual applications of $\mathrm{N}$ and $\mathrm{P}$ on corn grain yieds and soil chemical properties. Agron J 75, 94-99

Ketcheson JW (1980) Long-range effects of intensive cultivation and monoculture on the quality of southern Ontario soils. Can J Soil Sci 60, 403-410

Lubet E, Juste C (1979) Effets de l'introduction d'une prairie temporaire, d'un engrais vert et de l'exportation des résidus de récolte sur les monocultures de maïs implantées dans les sols sablo-limoneux du sud des Landes. CR Acad Agr Fr, 295-309

Martinez J, Guiraud G (1990) A lysimeter study of the effects of a rye-grass catch crop during a winter on wheat-maize rotation, on nitrate leaching and on the following crop. J Soil Sci 41, 5-16

Monnier $G$ (1965) Action des matières organiques sur la stabilité structurale des sols. Thèse Dr-Ing, Paris, $140 \mathrm{p}$

Page JB, Willard CJ (1946) Cropping systems and soil properties. Soil Sci Soc Proc 11, 81-88

Scott TW, Pleasant JM, Burt RF, Otis DJ (1987) Contributions of ground cover, dry matter, and nitrogen from intercrops and cover crops in a corn polyculture system. Agron J 79, 792-798

Snider HJ (1956) A successful corn crop on the same land every year is a possibility. Better Crops 40 , 13-14

Wagger MG (1989a) Time of dessication effects on plant composition and subsequent nitrogen release from several winter annual cover crops. Agron $J$ 81, 236-241

Wagger MG (1989b) Cover crop management and nitrogen rate in relation to growth and yield of no-till corn. Agron J 81, 533-538

Wagger MG, Mengel DB (1987) The role of nonleguminous cover crops in the efficient use of water and nitrogen. In: Cropping strategies for efficient use of water and nitrogen (WL Hargrove, ed) 115-127. Spec publ 51. Am Soc Agron, Madison, USA 